\title{
Genetic Modification of the Lactation Curve by Bending the Eigenvectors of the Additive Genetic Random Regression Coefficient Matrix
}

\author{
K. Togashi ${ }^{1}$ and C. Y. Lin† \\ *National Agricultural Research Centre in Hokkaido Region, Hitsujigaoka 1, Toyohiraku, Sapporo, Japan, 0628555 \\ †Dairy and Swine Research and Development Centre, Agriculture and Agri-Food Canada, Lennoxville, Québec, Canada J1M 1J3
}

\begin{abstract}
The eigenvectors of the additive genetic random regression covariance $(\mathbf{K})$ matrix contribute differentially to different parts of the lactation curve in response to genetic selection. It is, therefore, important to examine the genetic response patterns from the individual eigenvectors of the matrix $\mathbf{K}$ for the modification of the shape of the lactation curve. This study demonstrated a general methodology for imposing differential restrictions on different eigenvectors according to their effects on the shape of the lactation curve. A numerical example is given to illustrate the derivation and implementation of this procedure. Theoretically and experimentally, manipulating individual eigenvectors based on their individual effects on the shape of the lactation curve is more important than manipulating the joint effect of all the eigenvectors of $\mathbf{K}$ on the lactation curve. This described procedure provides a useful tool for simultaneous improvement of milk production and lactation persistency by modifying the shape of the lactation curve.
\end{abstract}

Key words: lactation curve, lactation milk, persistency, eigen index

\section{INTRODUCTION}

Persistency refers to the rate of decline in daily yield after the peak within lactation. A cow with a lower rate of decline (i.e., a higher persistency) makes better use of inexpensive forage (Solkner and Fuchs, 1987), suffers less stress from high peak yield (Zimmermann and Sommer, 1973; Muir et al., 2004; Weller et al., 2006), is more resistant to disease (Jakobsen, 2000; Harder et al., 2006), and is more profitable (Dekkers et al., 1998). Therefore, the modification of the lactation curve to increase lactational milk and persistency is economi-

Received May 13, 2007.

Accepted August 19, 2007.

${ }^{1}$ Corresponding author: tkenji@naro.affrc.go.jp cally important to the dairy industry and, thus, has garnered extensive investigation by animal scientists (Ferris et al., 1985; Gengler, 1996; Jamrozik et al., 1997; Swalve and Gengler, 1999).

The procedures for simultaneously improving lactational milk yield and persistency or for maximizing lactational milk yield subject to zero restriction in lactational persistency have been developed (Togashi and Lin, 2004; Lin and Togashi, 2005). Various studies have pointed out the possible use of the eigenvectors of the additive genetic random regression (RR) covariance matrix (K) or test-day additive genetic covariance matrix $(\mathbf{G})$ to increase lactational milk yield and persistency (Olori et al., 1999; Druet et al., 2003; Macciotta et al., 2004). These studies found that the first (leading) eigenvector was responsible for constant increase in milk yields across lactation, whereas the second eigenvector was related to the change in the shape of the lactation curve. All of these studies examined the relationship of the eigenvectors to the lactation curve on a population basis and, thus, cannot be used for selection decisions. The eigenvectors of the additive genetic $R R$ regression covariance have been used to reduce the computational requirements for genetic evaluation (Druet et al., 2003). Meyer (2006) used the eigenvectors of the multivariate covariance matrix to construct principal components to reduce the computational requirements so that larger data sets and more traits could be analyzed. Togashi and Lin $(2006,2007)$ constructed eigen indexes to quantify the relationship of individual eigenvectors of the matrix $\mathbf{K}$ to the genetic response and developed the unrestricted and restricted indexes to change the joint effect of the eigenvectors of the genetic covariance matrix $\mathbf{G}$ on the lactation curve. Those reports indicated that the eigenvectors of the covariance matrices provide a powerful, useful tool for parameter estimation, genetic evaluation, and development of selection criteria for achieving specific breeding goals.

The purpose of this study is to present a method to simultaneously improve lactational milk yield and persistency by modifying the specific effects of individ- 
ual eigenvectors on the lactation curve rather than changing the joint effect of the eigenvectors on the lactation curve.

\section{MATERIALS AND METHODS}

\section{Unrestricted Full Eigen Indexes of Matrix K}

Assume that test-day data were fitted with Legendre polynomial RR of degree $(k-1)$. Let $\alpha$ be a $(k \times 1)$ vector containing the additive genetic RR coefficients $\alpha=$ $\left[\begin{array}{llll}\alpha_{0} & \alpha_{1} & \cdots & \alpha_{k-1}\end{array}\right]^{\prime}$. The variance of $\alpha$ is a $(k \times k)$ additive genetic $R R$ covariance matrix $K$. The genetic covariance matrix of daily yields from DIM 5 to 305 is $\mathbf{G}=\boldsymbol{\Phi} \mathbf{K} \boldsymbol{\Phi}^{\prime}$, where $\boldsymbol{\Phi}=$ a $(301 \times k)$ matrix of Legendre polynomial coefficients (i.e., covariates) evaluated at DIM 5 through 305. Togashi and Lin (2006) constructed the unrestricted full eigen index $\left(\mathbf{I}_{\mathbf{U}}\right)$ as follows:

$$
\mathbf{I}_{\mathbf{U}}=\sum_{i=1}^{k} b_{i}\left(\alpha^{\prime} \mathbf{e}_{i}\right)=b^{\prime} \mathbf{E}^{\prime} \alpha
$$

where $b=\mathrm{a}(k \times 1)$ vector of index coefficients and $\mathbf{E}=$ $\left[\begin{array}{llll}e_{1} & e_{2} & \cdots & e_{k}\end{array}\right]$ with $\boldsymbol{e}_{i}$ being the normalized eigenvectors of $\mathbf{K}$ corresponding to eigen values, $\lambda_{i}$, that are sorted in descending order. Because an index trait arises exclusively from a specific eigenvector, the expression of an index trait is due exclusively to that eigenvector. The variance of $\mathbf{I}_{\mathbf{U}}$ is $\sigma_{1}^{2}=b^{\prime} \mathbf{E}^{\prime} \mathbf{K E} b=b^{\prime} \mathbf{D} b$, where $\mathbf{D}=\mathrm{a}(k \times k)$ diagonal matrix with eigen values of $\mathbf{K}$ on the diagonal. The covariance matrix of the index traits is $\operatorname{Var}\left(\mathbf{E}^{\prime} \alpha\right)=\mathbf{D}$, and the sum of the variances of all index traits is $\mathbf{1}^{\prime} \mathbf{D} \mathbf{1}=\sum_{i=1}^{k} \lambda_{i}$, with $\mathbf{1}$ being a vector of ones to create a sum.

Let the net merit $(\mathbf{H})$ be the genetic value of the lactation milk (i.e., $\left.\mathbf{H}=\mathbf{1}^{\prime} \mathbf{g}=\mathbf{1}^{\prime} \boldsymbol{\Phi} \alpha\right)$, where $\mathbf{g}=\mathrm{a}(301 \times \mathbf{1})$ vector containing the daily genetic values ranging from $\mathrm{d} 5$ to 305 . The correlation between the index $\mathbf{I}_{\mathbf{U}}=b^{\prime} \mathbf{E}^{\prime} \alpha$ and the net merit $\mathbf{H}=\mathbf{1}^{\prime} \boldsymbol{\Phi} \alpha$ is maximized when $b=\mathbf{D}^{-}$ ${ }^{1} \mathbf{E}^{\prime} \mathbf{K} \boldsymbol{\Phi}^{\prime} \mathbf{1}$ (Togashi and Lin, 2006). The genetic response for the $i$ th day of lactation to selection on $\mathbf{I}_{\mathbf{U}}$ is:

$$
\Delta G_{i}=b_{g_{i} \mathrm{I}_{\mathrm{U}}}(\mathrm{S})=\frac{\operatorname{Cov}\left(\boldsymbol{\Phi}_{i} \alpha, b^{\prime} \mathbf{E}^{\prime} \alpha\right)}{\sigma_{\mathrm{I}_{\mathrm{U}}}^{2}}(\mathbf{S})=\boldsymbol{\Phi}_{i} \mathbf{K E} b\left(\bar{i} / \sigma_{\mathrm{I}_{\mathrm{U}}}\right)
$$

where $\mathrm{S}=$ selection differential; $\bar{i}$ is selection intensity; and $\boldsymbol{\Phi}_{i}(I=5$ to 305$)$ is the $i$ th row of $\boldsymbol{\Phi}$. The genetic responses $(\Delta)$ for each day of lactation to selection on $\mathbf{I}_{\mathbf{U}}$ is:

$$
\underline{\Delta}=\frac{\operatorname{Cov}\left(\Phi \alpha, b^{\prime} \mathbf{E}^{\prime} \alpha\right)}{\sigma_{\mathrm{I}_{\mathrm{U}}}^{2}}(\mathrm{SD})=\boldsymbol{\Phi} \mathbf{K E} b\left(\bar{i} / \sigma_{\mathrm{I}_{\mathrm{U}}}\right)
$$

where $\underline{\Delta}=\left[\begin{array}{llll}\Delta G_{5} & \Delta G_{6} & \cdots & \Delta G_{305}\end{array}\right]^{\prime}$ and $\sigma_{\mathrm{I}_{\mathrm{U}}}^{2}=b^{\prime} \mathbf{D} b$.

\section{Individual Eigen Indexes of the Matrix K}

The index derived from the $i$ th eigenvector (called the $i$ th eigen index hereafter) is $\mathbf{I}_{(i)}=\alpha^{\prime} \boldsymbol{e}_{i}$, where $i$ ranges from 1 to $k$. The genetic responses for each day of lactation from selection on the $i$ th eigen index are:

$$
\underline{\Delta}^{(i)}=\frac{\operatorname{Cov}\left(\boldsymbol{\Phi} \alpha, \mathrm{I}_{(i)}\right)}{\sigma_{I_{(i)}}^{2}}(\mathrm{SD})=\boldsymbol{\Phi} \mathbf{K} \boldsymbol{e}_{i}\left(\frac{\bar{i}}{\sigma_{I_{(i)}}}\right),
$$

where $\Delta^{(i)}=\left[\begin{array}{lllll}\Delta G_{5}^{(i)} & \Delta G_{6}^{(i)} & \Delta G_{7}^{(i)} & \cdots \cdots & \Delta G_{305}^{(i)}\end{array}\right]^{\prime}$, with superscript $(i)$ denoting the $i$ th eigenvector and $\sigma_{\mathrm{I}_{(i)}}^{2}=$ $\mathbf{e}_{i}^{\prime} \mathbf{K} \mathbf{e}_{i}=\lambda_{i}$.

Because the eigenvectors of $\mathbf{K}$ are orthogonal, selection based on the $i$ th eigen index (i.e., the $i$ th index trait) produces no correlated responses in other index traits. According to [2], the genetic responses for each day of lactation $(\underline{\Delta})$ to selection on $\mathbf{I}_{\mathbf{U}}$ are,

$$
\begin{aligned}
& \underline{\Delta}=\boldsymbol{\Phi} \mathbf{K}\left[e_{1} e_{2} \quad \cdots \quad e_{k}\right] b\left(\bar{i} / \sigma_{\mathrm{I}_{\mathrm{U}}}\right)= \\
& \left(\boldsymbol{\Phi} \mathbf{K} e_{1} b_{1}+\Phi \mathbf{K} e_{2} b_{2}+\cdots+\Phi \mathbf{K} e_{k} b_{k}\right)\left(\bar{i} / \sigma_{\mathrm{I}_{\mathrm{U}}}\right)= \\
& \underline{\Delta}^{(1)}+\underline{\Delta}^{(2)}+\cdots+\underline{\Delta}^{(k)}
\end{aligned}
$$

where $\Delta^{(i)}=\mathrm{a}(301 \times 1)$ vector of the daily genetic responses attributed to the $i$ th eigenvector. Particularly, equation [4] looks as follows:

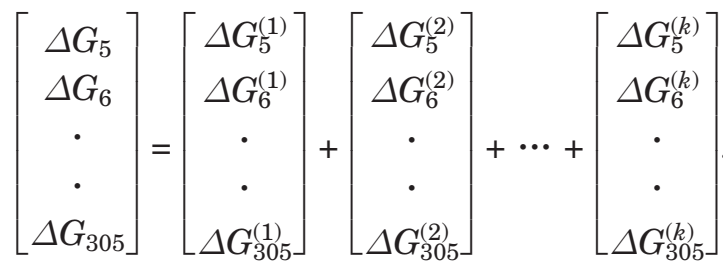

The left-hand side of this equation is the joint effect of all eigenvectors on the daily genetic responses across lactation, whereas the right-hand side is the sum of specific effects of individual eigenvectors on the daily genetic responses across lactation. Thus, vector $\Delta$ for all $k$ eigenvectors combined was partitioned into $k$ components, each representing the effect of an individual eigenvector (orthogonal decomposition). 


\section{Rationale Behind Bending the Individual Eigenvectors of Matrix $K$}

Togashi and Lin (2006) showed that individual eigenvectors of the matrix $\mathbf{K}$ contributed differentially to the shape of the lactation curve. Therefore, it is necessary to modify the pattern of genetic responses due to the individual eigenvectors of the matrix $\mathbf{K}$ to achieve the desired lactation curve. The modification requires a restricted selection index approach. Suppose we want to impose 2 sets of restrictions (denoted by vectors $u$ and $v$ ) on the daily genetic responses to the $i$ th and $j$ th eigenvectors of $\mathbf{K}$, respectively. The vectors $u$ and $v$ have the length of $n_{u}$ and $n_{v}$ containing the predetermined restrictions, where $n_{u}$ and $n_{v}$ refer to the total number of daily genetic gains restricted. Vectors $u$ and $v$ may or may not be the same and may contain negative, zero, or positive values. As an example, if the respective effects of the $i$ th and $j$ th eigenvectors on peak yield are restricted to zero, then $u=0$ and $v=0$.

Let the restricted eigen index $\mathbf{I}^{*}=b^{* \prime} \mathbf{E}^{\prime} \alpha$ be designed to maximize the lactation response while at the same time flattening the daily genetic responses from DIM 55 to 280 due to the $i$ th and the $j$ th eigenvector $\left(e_{i}\right.$ and $e_{j}$ ). According to [4], index $\mathbf{I}^{*}$ needs to meet 2 sets of restrictions: $\Phi^{*} \mathbf{K} e_{i} b_{i}{ }_{i}=\theta u$ and $\boldsymbol{\Phi}^{*} \mathbf{K} e_{j} b^{*}{ }_{j}=\xi v$, where $\boldsymbol{\Phi}^{*}=$ a (226×k) submatrix of $\Phi$ with rows 1 to 50 (corresponding to DIM 5 to 54) and rows 277 to 301 (corresponding to DIM 281 to 305 ) being deleted, because daily yields within these ranges were unrestricted. The Lagrange multipliers function takes the following form:

$$
\begin{gathered}
f=\operatorname{Var}\left(\mathbf{I}^{*}-\mathbf{H}\right)+\eta^{\prime}\left(\boldsymbol{\Phi}^{*} \mathbf{K} e_{i} b_{i}{ }_{i}-\theta u\right) \\
+\delta^{\prime}\left(\boldsymbol{\Phi}^{*} \mathbf{K} e_{j} b_{j}^{*}-\xi v\right)=b^{* \prime} \mathbf{D} b^{*}-2 b^{* \prime} \mathbf{E}^{\prime} \mathbf{K} \boldsymbol{\Phi}^{\prime} \mathbf{1} \\
+\mathbf{1}^{\prime} \mathbf{G} \mathbf{1}+\eta^{\prime}\left(\boldsymbol{\Phi}^{*} \mathbf{K} e_{i} b_{i}^{*}-\theta u\right)+\delta^{\prime}\left(\boldsymbol{\Phi}^{*} \mathbf{K} e_{j} b_{j}^{*}-\xi v\right),
\end{gathered}
$$

where $\eta$ and $\delta=$ the $(226 \times k)$ vectors of Lagrange multipliers, respectively, and $\theta$ and $\xi=$ scalars to be determined a posteriori. Taking the derivative of the function $f$ with respect to $b^{*}, \eta, \theta, \delta$, and $\xi$ and setting these partial derivatives equal to zeros results in the following:

$$
\begin{gathered}
\frac{\partial f}{\partial b^{*}}= \\
2 \mathbf{D} b^{*}-2 \mathbf{E}^{\prime} \mathbf{K} \Phi^{\prime} \mathbf{1}+\mathbf{E}_{i}^{\prime} \mathbf{K} \Phi^{* \prime} \eta+\mathbf{E}_{j}^{\prime} \mathbf{K} \Phi^{* \prime} \delta=0 \\
\frac{\partial f}{\partial \eta}=\Phi^{*} \mathbf{K} e_{i} b_{i}^{*}-\theta k_{i}=0 \\
\frac{\partial f}{\partial \theta}=-u^{\prime} \eta=0
\end{gathered}
$$

$$
\begin{gathered}
\frac{\partial f}{\partial \delta}=\boldsymbol{\Phi} * \mathbf{K} e_{j} b_{j}^{*}-\xi k_{j}=0 \\
\frac{\partial f}{\partial \xi}=-v^{\prime} \delta=0 .
\end{gathered}
$$

In equation [5], $\mathbf{E}_{i}$ and $\mathbf{E}_{j}=$ the $(k \times k)$ null matrices, with columns $i$ and $j$ being replaced by $e_{i}$ and $e_{j}$, respectively. Equations [6] and [8] can be rewritten as $\boldsymbol{\Phi}^{*} \mathbf{K E}_{i} b^{*}-\theta u=0$ and $\boldsymbol{\Phi}^{*} \mathbf{K E}_{j} b^{*}-\xi k v=0$, respectively.

The above 5 sets of equations can be expressed jointly in the following form:

$$
\begin{gathered}
{\left[\begin{array}{ccccc}
2 \mathbf{D} & \mathbf{E}_{i}^{\prime} \mathbf{K} \boldsymbol{\Phi}^{* \prime} & 0 & \mathbf{E}_{j}^{\prime} \mathbf{K} \boldsymbol{\Phi}^{* \prime} & 0 \\
\boldsymbol{\Phi}^{*} \mathbf{K} \mathbf{E}_{i} & 0 & -u & 0 & 0 \\
0 & -u^{\prime} & 0 & 0 & 0 \\
\boldsymbol{\Phi}^{*} \mathbf{K} \mathbf{E}_{j} & 0 & 0 & 0 & -v \\
0 & 0 & 0 & -v^{\prime} & 0
\end{array}\right]\left[\begin{array}{c}
b^{*} \\
\eta \\
\theta \\
\delta \\
\xi
\end{array}\right]=} \\
{\left[\begin{array}{c}
2 \mathbf{E}^{\prime} \mathbf{K} \boldsymbol{\Phi}^{\prime} \mathbf{1} \\
0 \\
0 \\
0 \\
0
\end{array}\right] .}
\end{gathered}
$$

The solution $b^{*}$ to equation [10] is expected to maximize lactational milk yield and satisfy the restrictions imposed on the $i$ th and $j$ th eigenvector. The genetic responses $\left(\Delta^{*}\right)$ in daily yields of the lactation due to selection on $\mathbf{I}^{*}$ are:

$$
\Delta^{*}=\boldsymbol{\Phi} \mathbf{K E} b^{*}\left(\bar{i} / \sigma_{I^{*}}\right)
$$

where $\sigma_{I^{*}}^{2}=b^{* \prime} \mathbf{E}^{\prime} \mathbf{K} \mathbf{E} b^{*}=b^{* \prime} \mathbf{D} b^{*}$.

\section{Restricting the Joint Effect of All Eigenvectors of Matrix $K$}

This section demonstrates how to impose restrictions on the joint effect of all the eigenvectors of $\mathbf{K}$. Let the restricted index $\left(\mathbf{I}_{\mathbf{j}}=b^{0^{\prime}} \mathbf{E}^{\prime} \alpha\right)$ be designed to maximize net merit $\mathbf{H}$ while restricting the joint effect of all the eigenvectors of $\mathbf{K}$. The restriction vector $k_{0}$ imposed is predetermined in the same way as the restriction vectors $u$ and $v$ above. The subscript of $\mathbf{I}_{\mathbf{j}}$ indicates the restriction on the joint effect $(\mathrm{j}=$ joint $)$. The index $\mathbf{I}_{\mathbf{j}}$ needs to meet the restriction of $\boldsymbol{\Phi}^{*} \mathbf{K E} b^{\circ}=\theta k_{0}$. The Lagrange multipliers function takes the following form:

$$
\begin{gathered}
f=\operatorname{Var}\left(\mathbf{I}_{\mathbf{j}}-\mathbf{H}\right)+\eta^{\prime}\left(\boldsymbol{\Phi}^{*} \mathbf{K E} b^{\circ}-\theta k_{0}\right)= \\
b^{\circ \prime} \mathbf{D} b^{\circ}-2 b^{\circ} \mathbf{E}^{\prime} \mathbf{K} \boldsymbol{\Phi}^{\prime} \mathbf{1}+\mathbf{1}^{\prime} \mathbf{G} \mathbf{1}+\eta^{\prime}\left(\boldsymbol{\Phi}^{*} \mathbf{K} \mathbf{E} b^{\circ}-\theta k_{0}\right) .
\end{gathered}
$$


Taking the derivative of the function $f$ with respect to $b^{\circ}, \eta$, and $\theta$ and equating these partial derivatives to zeros result in the following set of equations:

$$
\begin{gathered}
\frac{\partial f}{\partial b^{\circ}}=2 \mathbf{D} b^{\circ}-2 \mathbf{E}^{\prime} \mathbf{K} \boldsymbol{\Phi}^{\prime} \mathbf{1}+\mathbf{E}^{\prime} \mathbf{K} \boldsymbol{\Phi}^{* \prime} \eta=0 \\
\frac{\partial f}{\partial \eta}=\boldsymbol{\Phi}^{*} \mathbf{K} \mathbf{E} b^{\circ}-\theta k_{0}=0 \\
\frac{\partial f}{\partial \theta}=-k_{0}^{\prime} \eta=0 .
\end{gathered}
$$

Thus, the index that satisfies the restrictions can be obtained from the following equations:

$$
\left[\begin{array}{ccc}
2 \mathbf{D} & \mathbf{E}^{\prime} \mathbf{K} \boldsymbol{\Phi}^{* \prime} & 0 \\
\boldsymbol{\Phi}^{*} \mathbf{K E} & 0 & -k_{0} \\
0 & -k_{0}^{\prime} & 0
\end{array}\right]\left[\begin{array}{c}
b^{\circ} \\
\eta \\
\theta
\end{array}\right]=\left[\begin{array}{c}
2 \mathbf{E}^{\prime} \mathbf{K} \boldsymbol{\Phi}^{\prime} \mathbf{1} \\
0 \\
0
\end{array}\right] .
$$

The matrix $\mathbf{E}$ in equation [12] contains all the eigenvectors of $\mathbf{K}$, due to restriction on the joint effect of all the eigenvectors of $\mathbf{K}$. In contrast, the matrices $\mathbf{E}_{i}$ and $\mathbf{E}_{j}$ in equation [10] contain the $i$ th and $j$ th eigenvectors, respectively, due to the restriction on eigenvectors $i$ and $j$.

\section{Numerical Example}

The matrices $\mathbf{K}, \mathbf{E}, \mathbf{D}$, and $\boldsymbol{\Phi}$ (Togashi and Lin, 2006) were used for this example. These matrices were derived from the analysis of the first lactation milk of Japanese Holstein cows using a RR animal model fitted with a quartic $(k=5)$ Legendre polynomial (Togashi et al., 2005). Thus, the number of index traits is 5. To reduce the number of the restricted eigen index equations, the first lactation was grouped into 9 stages: stage 1 ranges from DIM 5 to 60 , stages 2 to 8 have an interval of $30 \mathrm{~d}$ each, and stage 9 ranges from DIM 271 to 305.

Index with Proportional Restriction on the Effect of the Third Eigenvector. Togashi and Lin (2006) reported that the first eigenvector of $\mathbf{K}$ is responsible for raising the production level without altering the shape of the lactation curve, the second eigenvector accounts for increasing trend in daily genetic responses from DIM 5 to 301, and the daily genetic responses due to the third eigenvector is a concave curve. Therefore, the effects of the first and second eigenvectors on the lactation curve are desirable with respect to persistency, whereas the effect of the third eigenvector is undesirable. It makes sense to modify the undesirable effect of the third eigenvector on persistency. One possible way of achieving this purpose is to derive an eigen index $\left(\mathbf{I}_{3}\right)$ subject to the restriction that the genetic responses for stages 2 to 8 from the third eigenvector be equal $\left(\Delta G_{s 2}^{\mathbf{I}_{3}}=\Delta G_{s 3}^{\mathbf{I}_{3}}=\cdots=\Delta G_{s 8}^{\mathbf{I}_{3}}\right)$. The imposition of equal genetic responses from stages 2 to 8 (a restriction of 7 stages) means that the restriction vector $u$ has an order of 7: $u=\left[\begin{array}{lllllll}100 & 100 & 100 & 100 & 100 & 100 & 100\end{array}\right]^{\prime}$.

Because the restriction is imposed on the third eigenvector alone, equation [10] reduces to:

$$
\left[\begin{array}{ccc}
2 \mathbf{D} & \mathbf{E}_{3}^{\prime} \mathbf{K} \boldsymbol{\Phi}^{* \prime} & 0 \\
\boldsymbol{\Phi}^{*} \mathbf{K} \mathbf{E}_{\mathbf{3}} & 0 & -u \\
0 & -u^{\prime} & 0
\end{array}\right]\left[\begin{array}{c}
b^{*} \\
\eta \\
\theta
\end{array}\right]=\left[\begin{array}{c}
2 \mathbf{E}^{\prime} \mathbf{K} \boldsymbol{\Phi}^{\prime} \mathbf{1} \\
0 \\
0
\end{array}\right] .
$$

The matrix $\Phi^{*}$ has a dimension of $7 \times 5$ corresponding to stages 2 to 8 and contains the sum of each order of Legendre polynomials within each of these stages.

$$
\boldsymbol{\Phi}^{*}=\left[\begin{array}{ccccc}
21.213 & -19.473 & -3.493 & 22.987 & -20.429 \\
21.213 & -12.125 & -15.732 & 22.277 & 0.995 \\
21.213 & -4.776 & -22.278 & 10.454 & 19.216 \\
21.213 & 2.572 & -23.132 & -5.747 & 21.940 \\
21.213 & 9.920 & -18.293 & -19.590 & 7.563 \\
21.213 & 17.269 & -7.763 & -24.342 & -14.827 \\
21.213 & 24.617 & 8.460 & -13.266 & -25.451
\end{array}\right]
$$

The matrix $\mathbf{E}_{\mathbf{3}}$ is obtained from the matrix $\mathbf{E}$ by setting the unrestricted eigenvectors to zero.

$$
\mathbf{E}_{3}=\left[\begin{array}{cccccc}
0 & 0 & 0.0667 & 0 & 0 \\
0 & 0 & 0.1472 & 0 & 0 \\
0 & 0 & 0.9829 & 0 & 0 \\
0 & 0 & -0.0122 & 0 & 0 \\
0 & 0 & 0.0879 & 0 & 0
\end{array}\right]
$$

Substituting the matrices $\mathbf{K}, \mathbf{D}, \mathbf{E}, \mathbf{E}_{\mathbf{3}}, \boldsymbol{\Phi}$, and $\Phi^{*}$ and vector $u$ into [13] gives the following eigen index with equal proportional restriction:

$$
\mathbf{b}^{*}=\left[\begin{array}{lllll}
211.46 & -16.37 & 61.36 & -8.44 & -0.757
\end{array}\right]
$$

Therefore, $\mathbf{I}_{\mathbf{3}}=211.46 \mathrm{x}_{1}-16.37 \mathrm{x}_{2}+61.36 \mathrm{x}_{3}-8.44 \mathrm{x}_{4}$ $-0.757 \mathrm{x}_{5}$, where $\mathrm{x}_{1}=\mathbf{e}_{1}^{\prime} \boldsymbol{\alpha}, \mathrm{x}_{2}=\mathbf{e}_{2}^{\prime} \boldsymbol{\alpha}, \mathrm{x}_{3}=\mathbf{e}_{3}^{\prime} \boldsymbol{\alpha}, \mathrm{x}_{4}=$ $\mathbf{e}_{4}^{\prime} \boldsymbol{\alpha}$, and $\mathrm{x}_{5}=\mathbf{e}_{5}^{\prime} \boldsymbol{\alpha}$, with $\mathbf{e}_{\mathrm{i}}$ being the ith eigenvector of $\mathbf{K}$ and $\boldsymbol{\alpha}=\left(\begin{array}{lllll}\alpha_{0} & \alpha_{1} & \alpha_{2} & \alpha_{3} & \alpha_{4}\end{array}\right)^{\prime}$ being the additive genetic RR coefficients of each animal.

Index with Proportional Restriction on the Joint Effect of All Eigenvectors. This restricted eigen index denoted as $\mathbf{I}_{\mathbf{j}}$ was designed to maximize lactation milk with a restriction that the genetic responses for stages 2 to 8 due to $\mathbf{I}_{\mathbf{j}}$ be all the same $\left(\Delta G_{s 2}^{\mathbf{I}_{\mathbf{j}}}=\Delta G_{s 3}^{\mathbf{I}_{\mathbf{j}}}=\cdots=\right.$ 
$\Delta G_{\mathrm{s} 8}^{\mathbf{j}_{\mathbf{j}}}$. The desired index $\mathbf{I}_{\mathbf{j}}$ was obtained from equation [12] by setting the restriction vector $k_{0}$ to be: $k_{0}=$ $\left[\begin{array}{lllllll}100 & 100 & 100 & 100 & 100 & 100 & 100\end{array}\right]$.

Substituting the matrices $\mathbf{K}, \mathbf{D}, \mathbf{E}, \boldsymbol{\Phi}$, and vector $k_{0}$ into [12] gives the restricted eigen index $\mathbf{I}_{\mathbf{j}}$ :

$$
\mathbf{I}_{\mathbf{j}}=74.55 \mathrm{x}_{1}-5.97 \mathrm{x}_{2}-51.31 \mathrm{x}_{3}-7.94 \mathrm{x}_{4}+20.20 \mathrm{x}_{5},
$$

where the original solution $b^{0}$ has been divided by 100 without affecting the ranking of the animals, and variables $\mathrm{x}_{1}$ to $\mathrm{x}_{5}$ are as defined for the restricted eigen index $\mathbf{I}_{3}$. The genetic responses of daily yields from DIM 5 to 305 due to $\mathbf{I}_{\mathbf{3}}$ and $\mathbf{I}_{\mathbf{j}}$ were computed according to Togashi and Lin (2006).

\section{RESULTS AND DISCUSSION}

As shown above, the derivation of the unrestricted or restricted eigen index is a 2-step procedure: (1) apply principal component analysis (Holland, 1969; Johnson and Wichern, 1982) to derive the eigen or index traits (known as principal components) and (2) apply the index theory to maximize the correlation between the eigen index and the net merit to derive the eigen index coefficients. The application of principal component analysis depends solely upon the additive genetic $R R$ covariance matrix $\mathbf{K}$ and does not require a multivariate normal assumption. The vector $\alpha$ that contains a set of additive genetic $R R$ coefficients implicitly assumes that there is a linear relationship among those $\mathrm{RR}$ coefficients. Maximizing the correlation between the eigen index and net merit requires the assumption that the eigen traits and net merit follow a multivariate normal distribution. Furthermore, the realization of the expected genetic response requires the assumption of phenotypic-genetic parameters being estimated without error. The effect of the violation of these assumptions on the efficiency of the eigen index merits further research.

The pattern of daily genetic responses to the restricted eigen indexes $\mathbf{I}_{\mathbf{3}}$ and $\mathbf{I}_{\mathbf{j}}$ is in Figure 1. The index $\mathbf{I}_{3}$ produced a flatter curve of daily genetic responses than the index $\mathbf{I}_{\mathbf{j}}$, indicating that equal restriction on the effect of the third eigenvector is more effective than equal restriction on the joint effect of all the eigenvectors of $\mathbf{K}$ in terms of genetic improvement of persistency. This is because the index $\mathbf{I}_{3}$ takes into account the specific effect of the third eigenvector on the lactation curve, whereas the index $\mathbf{I}_{\mathbf{j}}$ fails to consider different characteristics of individual eigenvectors. Because each eigenvector contributes differentially to different parts of the lactation curve in response to selection, it is important to modify the effect of individual eigenvectors of $\mathbf{K}$ accordingly. The advantage of restricting individual

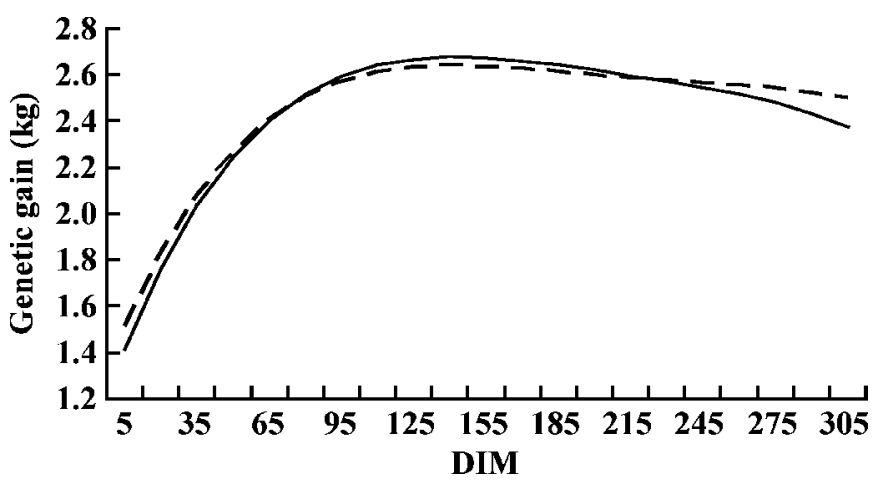

Figure 1. Daily genetic responses across lactation due to the index with joint restriction on all the eigenvectors $\left(\mathbf{I}_{\mathbf{j}} ;-\right)$ and to the index with restriction on the third eigenvector alone $\left(\mathbf{I}_{3} ;---\right)$.

effects of the eigenvectors over restricting the joint effect of all eigenvectors depends upon the net merit and the severity of the constraints.

Equation [10] can be reduced or augmented depending upon the number of eigenvectors restricted in combination or separately. It is a generalized set of equations for imposing single or multiple restriction(s) on the daily genetic responses to different eigenvectors. The desired index is expected to maximize net merit $(\mathbf{H})$ while meeting the restrictions imposed. The procedure presented provides a useful tool to manipulate individual eigenvectors according to their specific effects on the shape of the lactation curve. However, it is noteworthy that the restriction imposed should be biologically reasonable. Too severe of restrictions would require too high a selection intensity to sustain the population or may lead to the singularity of the covariance matrix of the restricted index equations.

\section{CONCLUSIONS}

Individual eigenvectors of the additive genetic $R R$ covariance matrix $\mathbf{K}$ contribute differentially to different parts of the lactation curve in response to selection. It is of importance to study the genetic effect of each of these eigenvectors on the shape of the lactation curve. Restricted index selection based on lactation EBV imposes restrictions on the collective effect of all the eigenvectors of $\mathbf{K}$ regardless of differential effects of individual eigenvectors on the shape of the lactation curve. This study demonstrated the theory and application of modifying the effects of specific eigenvectors on the shape of the lactation curve. Theoretically and experimentally, restricting specific effects of individual eigenvectors was preferable to restricting the joint effect of all the eigenvectors combined. It is justified to impose differential restrictions on different eigenvectors ac- 
cording to their effects on the shape of the lactation curve.

\section{REFERENCES}

Dekkers, J. C. M., J. H. Ten Haag, and A. Weersink. 1998. Economic aspects of persistency of lactation in dairy cattle. Livest. Prod. Sci. 53:237-252.

Druet, T., F. Jaffrézic, D. Boichard, and V. Ducrocq. 2003. Modeling lactation curves and estimation of genetic parameters for first lactation test-day records of French Holstein cows. J. Dairy Sci. 86:2480-2490.

Ferris, T. A., I. L. Mao, and C. R. Anderson. 1985. Selection for lactation curve and milk yield in dairy cattle. J. Dairy Sci. 68:1438-1448.

Gengler, N. 1996. Persistency of lactation yields: A review. Interbull Bull. 12:87-96.

Harder, B., J. Bennewitz, D. Hinrichs, and E. Kalm. 2006. Genetic parameters for health traits and their relationship to different persistency traits in German Holstein dairy cattle. J. Dairy Sci. 89:3202-3212.

Holland, D. A. 1969. Component analysis: An aid to the interpretation of data. Exp. Agric. 5:151-164.

Jakobsen, J. H. 2000. Genetic correlations between the shape of the lactation curve and disease resistance in diary cattle. $\mathrm{PhD}$ Thesis. Dept. Anim. Breed. Genet., Dan. Inst. Agric. Sci., Research Centre, Foulum, Denmark.

Jamrozik, J., L. R. Schaeffer, and J. C. M. Dekkers. 1997. Genetic evaluation of dairy cattle using test day yields and random regression model. J. Dairy Sci. 80:1217-1226.

Johnson, R. A., and D. W. Wichern. 1982. Applied Multivariate Statistical Analysis. Prentice-Hall Int. Inc., London, UK.

Lin, C. Y., and K. Togashi. 2005. Maximization of lactation milk production without decreasing persistency. J. Dairy Sci. 88:2975-2980.
Macciotta, N. P. P., D. Vicario, C. D. Mauro, and A. Cappio-Borlina. 2004. A multivariate approach to modeling shapes of individual lactation curves in cattle. J. Dairy Sci. 87:1092-1098.

Meyer, K. 2006. To have your steak and eat it: Genetic principal component analysis for beef cattle data. Proc. 8th WCGALP Commun. No. 25-03. 8th WCGALP Organizing Committee, Belo Horizonte, Brazil.

Muir, B. L., J. Fatehi, and L. R. Schaeffer. 2004. Genetic relationships between persistency and reproductive performance in first-lactation Canadian Holsteins. J. Dairy Sci. 87:3029-3037.

Olori, V. E., W. G. Hill, B. J. McGuirk, and S. Brotherstone. 1999. Estimating variance components for test-day milk records by restricted maximum likelihood with a random regression animal model. Livest. Prod. Sci. 61:53-63.

Solkner, J., and W. Fuchs. 1987. A comparison of different measures of persistency with special respect to variation of test-day milk yields. Livest. Prod. Sci. 16:305-319.

Swalve, H. H., and N. Gengler. 1999. Genetics of lactation persistency. Occ. Publ. Br. Soc. Anim. Sci. 24:75-82.

Togashi, K., Y. Atagi, J. Sato, T. Shirai, H. Takeda, and T. Yamazaki. 2005. Index selection for increased milk yield in mid and late lactation with the highest probability. Sustain. Livest. Prod. Human Welf. 59:877-881.

Togashi, K., and C. Y. Lin. 2004. Development of an optimal index to improve lactation yield and persistency with the least selection intensity. J. Dairy Sci. 87:3047-3052.

Togashi, K., and C. Y. Lin. 2006. Selection for milk production and persistency using eigenvectors of the random regression coefficient matrix. J. Dairy Sci. 89:4866-4873.

Togashi, K., and C. Y. Lin. 2007. Improvement of lactation milk and persistency using the eigenvectors of the genetic covariance matrix between lactation stages. Livest. Sci. 110:64-72

Zimmermann, E., and H. Sommer. 1973. Zum Laktationsverlauf von Kuhen in Hochleistung-sherden und dessen Beeinflussung durch nichterbliche Faktoren. Zuchtungskunde 45:75-88.

Weller, J. I., E. Ezra, and G. Leitner. 2006. Genetic analysis of persistency in the Israeli Holstein population by the multi-trait animal model. J. Dairy Sci. 89:2738-2746. 